\title{
Grain-size dependence of plastic deformation in nanocrystalline Fe
}

\author{
D. Jang \\ Department of Materials Science and Engineering, University of Michigan, Ann Arbor, Michigan 48109 \\ M. Atzmon ${ }^{\mathrm{a}}$ \\ Department of Materials Science and Engineering, University of Michigan, Ann Arbor, Michigan 48109 \\ and Department of Nuclear Engineering and Radiological Science, University of Michigan, \\ Ann Arbor, Michigan 48109
}

(Received 30 September 2002; accepted 28 February 2003)

\begin{abstract}
Plastic deformation of nanocrystalline $\mathrm{Fe}$ was investigated by nanoindentation. Samples, synthesized by mechanical attrition, consisted of powder particles with diameters greater than 30 $\mu \mathrm{m}$. The average grain diameters within the particles of different samples ranged from $10 \mathrm{~nm}$ to 10 $\mu \mathrm{m}$. To avoid potential artifacts, samples were prepared without use of heat treatment, and measurements were conducted at a depth significantly smaller than the powder particle size. Corrections were made for the indentation-size effect and for pileup or sink in around the indent. The volume-averaged grain size was used in the analysis. The Hall-Petch relation is obeyed for grain sizes above about $18 \mathrm{~nm}$, and slight softening occurs at smaller grain sizes. The strain-rate sensitivity increases monotonically with decreasing grain size. The results are consistent with grain-boundary sliding. (C) 2003 American Institute of Physics. [DOI: 10.1063/1.1569035]
\end{abstract}

\section{INTRODUCTION}

Nanocrystalline materials, reported by Gleiter, ${ }^{1}$ have been attracting much scientific and applied interest. Having grain sizes in the range of a few to tens of nanometers, they exhibit unusual properties. For example, their strength and hardness are reported to be significantly higher than those of conventional materials. ${ }^{2}$ Various synthesis methods have been developed, which include inert-gas condensation, ${ }^{3,4}$ mechanical attrition, ${ }^{5}$ and crystallization of amorphous materials. ${ }^{6}$ Mechanical attrition by ball milling is distinguished by its simplicity, low cost, and applicability to many metallic materials.

In conventional polycrystalline metals, the yield strength is known to increase with decreasing grain size. This behavior is often used to engineer hard materials. It has been explained as resulting from the larger stress required for dislocation pileup within a small grain. An experimental relationship between the grain size and yield strength of a material was obtained by $\mathrm{Hall}^{7}$ and by Petch: ${ }^{8}$

$$
\sigma_{y}=\sigma_{0}+\frac{k_{0}}{\sqrt{d}}
$$

where $d$ is the average grain size, $\sigma_{y}$ is the yield strength, $\sigma_{0}$ is the yield strength in a large-grained material, and $k_{0}$ is a material constant. This relation has attracted attention in the context of nanocrystalline materials, ${ }^{2,9}$ because of the high strength it predicts. Measuring the hardness, which is proportional to the yield strength, ${ }^{10}$ is less demanding with regard to sample geometry. The hardness value is the ratio of load to the resulting indentation area when pressing a standard indenter into a material. ${ }^{11}$ Experimental hardness data have

\footnotetext{
a) Author to whom correspondence should be addressed; electronic mail: atzmon@umich.edu
}

been controversial. There have been reports of a decrease in hardness at small grain sizes, ${ }^{12-21}$ termed "inverse HallPetch" behavior, but some of these results were affected by porosity or history of heat treatment. ${ }^{22}$ A review of past experimental reports is given in Ref. 23. Khan et al. ${ }^{21}$ measured the hardness dependence on the grain size in nanocrystalline Fe. They sintered powder at elevated temperatures to form bulk samples, and observed significant softening with decreasing grain size below $35 \mathrm{~nm}$. On the other hand, Malow and $\mathrm{Koch}^{24}$ observed the hardness to increase with decreasing grain size without exhibiting softening. We note that many studies suffer from an uncertainty in the grain size. Furthermore, the contribution of a grain to the sample hardness is proportional to the grain volume. Therefore, if the grain-size distribution is broad, it is important to use a volume-averaged grain size in the Hall-Petch plot.

In sufficiently small-grained nanocrystalline material, dislocations are believed to be absent. ${ }^{25,26}$ One would therefore expect a dominant deformation mechanism that is not based on dislocation pileups. This is consistent with observations reported by $\mathrm{Ke}$ et al. ${ }^{27}$ In this case, Eq. (1) is not expected to be applicable. Schiøtz et al. ${ }^{28}$ using moleculardynamic simulation, predicted softening of a polycrystalline material with decreasing grain size, when grain boundary sliding is the dominant deformation mechanism. Van Swygenhoven and Caro obtained a similar result. ${ }^{29}$ In this case, or for other thermally activated deformation mechanisms, the yield stress is expected to depend on the strain rate. One can define the strain-rate sensitivity as

$$
m=\frac{d \log \sigma}{d \log \dot{\varepsilon}}
$$

where $\sigma$ is the stress and $\dot{\varepsilon}$ is the strain rate. These are given by 


$$
\dot{\varepsilon}=\frac{1}{h} \frac{d h}{d t},
$$

and, for nanoindentation hardness measurements using a Berkovich tip,

$$
\sigma=\frac{L}{24.5 h^{2}},
$$

where $h$ is the displacement of the indenter and $L$ is the applied load. ${ }^{30}$ The strain-rate sensitivity, which is significant in coarse-grained materials only at high temperature, becomes of potential importance in nanocrystalline materials because their high grain boundary area may make timedependent deformation possible even at room temperature.

It is often difficult to eliminate experimental artifacts when attempting to measure the intrinsic hardness of a material. For example, bulk samples formed by compacting nanoparticles typically display significant porosity. ${ }^{24}$ In addition, controlling the grain size by thermal treatment often leads to a change in impurity distribution and therefore affects $\sigma_{0}$. However, the porosity effect can be overcome if the indentations used are sufficiently smaller than the particle size, so as to avoid a scale on which the material is porous. If the indentation size is also significantly smaller than the contact area between particles, then the particles can be considered well supported.

In this paper, nanoindentation hardness measurements in nanocrystalline $\mathrm{Fe}$, formed by mechanical attrition, are reported as a function of the volume-averaged grain size. An elemental system was selected to eliminate the influence of chemical effects. Annealing and porosity effects were eliminated by using as-milled samples and employing indentation sizes significantly smaller than the particle size, respectively. Tian and Atzmon ${ }^{31}$ have previously performed extensive studies of the grain size and size distribution in nanocrystalline $\mathrm{Fe}$, which provide useful input for the present study.

\section{EXPERIMENTAL PROCEDURE}

99.9\% pure Fe powder was obtained from the Cerac company, and all samples originated from the same batch. Nanocrystalline Fe was prepared at room temperature using a Fritsch Pulverisette-0 vibrating-frame ball mill with a hardened steel ball $5 \mathrm{~cm}$ in diameter. Prior to each run, the vial was evacuated to a pressure below $0.13 \mathrm{~Pa}$ and backfilled with purified argon. The vial vibration frequency was $50 \mathrm{~Hz}$, and the amplitude values used ranged from 1.5 to $1.8 \mathrm{~mm}$. Each data point was obtained from a sample milled without interruption, using $3.6 \mathrm{~g}$ of Fe powder. The powder particle sizes were observed by scanning electron microscopy (SEM) to be at least $30 \mu \mathrm{m}$ for all milled samples, and the indentation depth was less than $0.5 \mu \mathrm{m}$. The concentrations of $\mathrm{C}$ and $\mathrm{O}$ were measured by the LECO technique and determined to be less than $1.5 \mathrm{wt} \%$.

X-ray diffraction was performed using a horizontal General Electric $\theta-2 \theta$ powder diffractometer in step-scanning mode with Mo $K \alpha$ radiation $(\lambda=0.07093 \mathrm{~nm})$. The peaks were fitted to a Pearson VII function, and the volumeaveraged grain size was determined by using the integral

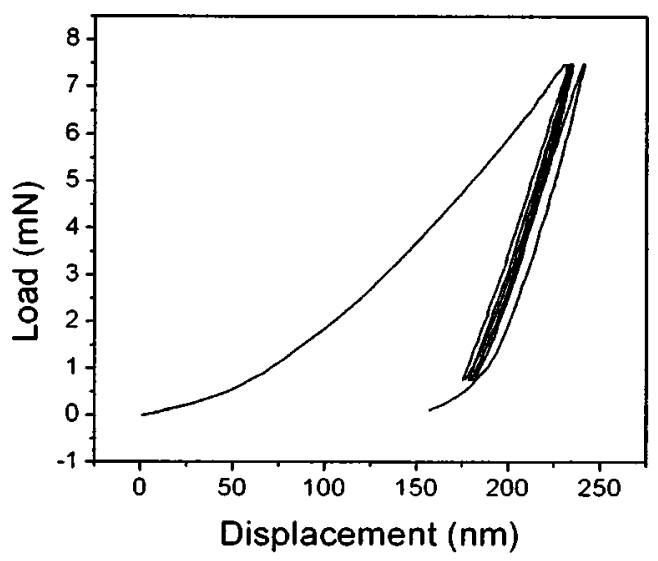

FIG. 1. Load vs indenter displacement measured in nanocrystalline Fe with a volume-averaged grain size of $10.5 \mathrm{~nm}$.

breadth and the data of Ref. 31, based on the WarrenAverbach analysis. To prepare samples for hardness measurements, the powder was pressed under $17 \mathrm{MPa}$ to form a bulk disk. Selected samples were subsequently further consolidated by plastic deformation using a cone-shaped plunger in a die. A smooth surface was obtained by mechanical polishing, using alumina paste with successively smaller particle sizes down to $0.05 \mu \mathrm{m}$. Attempts to chemically polish the samples were unsuccessful. However, it is not expected that mechanical polishing will introduce drastic changes in the already severely hardened samples. The hardness measurement was started within 60 min of polishing a sample, using a Nano II Nanoindenter with a Berkovich tip. The area function of the diamond tip and the load frame stiffness were calibrated using a silica standard. ${ }^{32}$ Two different sequence types were used. One was conducted at a fixed maximum load, i.e., all samples were indented to the same maximum load of $7.5 \mathrm{mN}$ at a rate of $0.75 \mathrm{mN} / \mathrm{s}$. Several sequences were conducted at a fixed maximum displacement each, ranging from 100 to $500 \mathrm{~nm}$, at a rate of $15 \mathrm{~nm} / \mathrm{s}$. For all measurements, loading and unloading was repeated three times, and a $100 \mathrm{~s}$ hold segment at $10 \%$ of the peak load was applied after the third unloading to correct for possible drift. Figure 1 shows a typical load vs displacement curve resulting from the loading sequence employed.

The indentations were inspected by SEM and atomicforce microscopy to verify the absence of cracks and determine the correct indentation area. ${ }^{33}$ In addition, data points were discarded for which the elastic modulus was significantly lower than the value for nanocrystalline Fe with 10 nm grain size, $\sim 195 \mathrm{GPa}^{34,35} 15-30$ data points were averaged to obtain each hardness value, and 15 data points for each value of $m$. The error was taken to be the standard deviation of the mean. It does not include potential systematic error in the extrapolation to infinite depth (see below).

\section{RESULTS AND DISCUSSION}

When using small indentations, the hardness appears larger than the bulk value. Nix and $\mathrm{Gao}^{36}$ corrected for this 


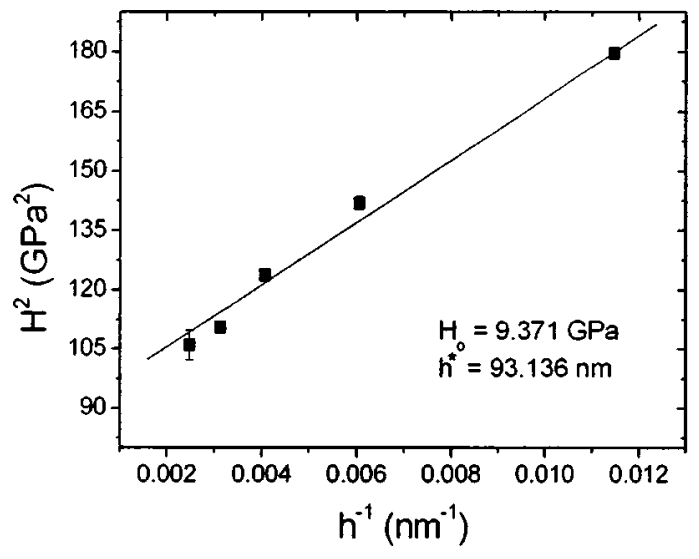

FIG. 2. Indentation-size effect: $H^{2}$ vs $h^{-1}$ plot for the determination of the hardness at the infinite depth for a sample with volume-averaged grain size of $18 \mathrm{~nm}$.

indentation-size effect for a dislocation mechanism of deformation. They obtained the following expression for the hardness $H$ :

$$
\frac{H(h)}{H_{0}}=\sqrt{1+h^{*} / h},
$$

where $h$ is the indentation depth, $H_{0}$ is the hardness in the limit of infinite depth, and $h^{*}$ is a characteristic length that depends on the shape of the indenter. While the model of Nix and Gao may be inapplicable to our samples, we use this expression here as a phenomenological fit to extrapolate our data to infinite depth. A typical fitting result is displayed in Fig. 2. Improved knowledge of the deformation mechanisms in nanocrystalline metals will be necessary to derive an expression for the indentation size effect. The results we present below are extrapolated values at infinite depth.

In Fig. 3, the hardness is plotted as a function $d^{-1 / 2}$, where $d$ is the volume-averaged grain size. The data presented were obtained at fixed maximum displacement, and corrected for the indentation area (from SEM) and indentation-size effect. We found that fixed-maximum-load and fixed-maximum-displacement data followed very similar

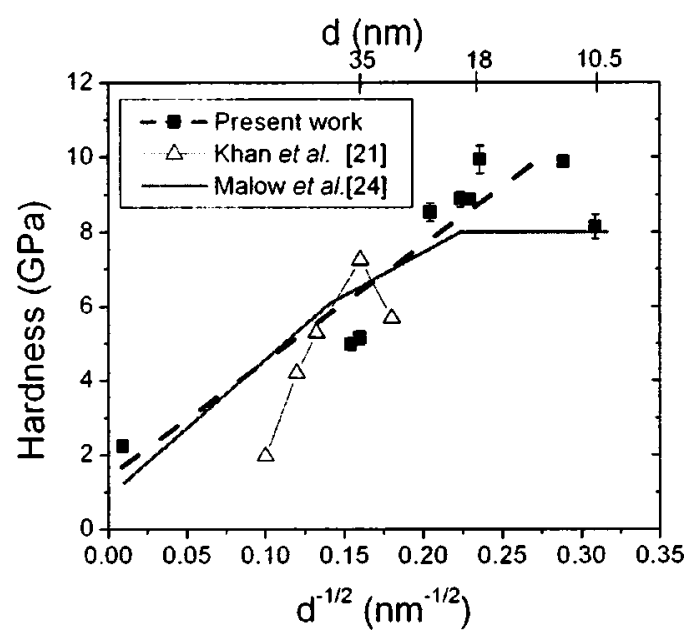

FIG. 3. Hardness of nanocrystalline Fe as a function of $d^{-1 / 2}$, where $d$ is the volume-averaged grain size. trends and displayed only a small systematic difference, indicating the absence of an artifact due to a surface layer. In addition, the thickness of the oxide surface layer was $4.2 \mathrm{~nm}$, as determined by Rutherford backscattering spectrometry. This value is negligibly small compared with the indentation depth. A possible additional artifact could be caused by an impurity concentration that correlates with the milling time and therefore with the grain size. However, LECO measurements of $\mathrm{C}$ and $\mathrm{O}$ concentrations displayed random sampleto-sample variations that did not correlate with the milling time. Therefore, we are confident that impurities do not contribute significantly to the behavior observed in Fig. 3. Additional compaction by further plastic deformation did not affect the results significantly, ruling out an artifact due to porosity.

The observed behavior for grain sizes greater than $18 \mathrm{~nm}$ is in agreement with the Hall-Petch relation. However, the slope decreases and becomes negative as the grain size decreases below $18 \mathrm{~nm}$. For comparison, data by Khan et al. ${ }^{21}$ and Malow and $\mathrm{Koch}^{24}$ are displayed together with the present data. The inverse square roots of the grain sizes of data in Refs. 21 and 24 are both based on the Scherrer equation $;{ }^{37}$ they were converted into volume-averaged values based on the extensive data of Ref. 31 , in order to obtain a meaningful comparison with the present data. The data of Ref. 21 display softening with decreasing grain size below $35 \mathrm{~nm}$. Since no softening is observed in this grain-size range in our data, we suggest that the data in Ref. 21 for grain sizes below $35 \mathrm{~nm}$ suffer from an artifact, likely due to porosity on a scale comparable to or smaller than the indentation size of $15 \mu \mathrm{m} .{ }^{24}$ We note that extrapolation of the Hall-Petch line presented in Ref. 21 shows a negative intercept with the ordinate, i.e., $\sigma_{0}$ in Eq. (1) is negative. This unphysical result is likely to be the result of a systematic error in the grain-size determination from diffraction peak widths in a range in which Scherrer broadening is small.

The data of Ref. 24 level off below $20 \mathrm{~nm}$, but with hardness values lower than in the present study. However, Ref. 24 does not contain data points between 10 and $20 \mathrm{~nm}$, where our data are the highest. We note that Malow and $\mathrm{Koch}^{24}$ also reported higher hardness values, $10 \mathrm{GPa}$, from a prior study. However, the grain-size data were later judged by the authors not to be reliable ${ }^{24}$ so we were not able to include them in Fig. 3. We suggest that the hardness decrease at small grain size is due to a transition to a dominant, thermally activated, deformation mechanism. This is consistent with the observations of Ke et al. ${ }^{27}$ who reported that nanocrystalline gold films with grain size of $10 \mathrm{~nm}$ deformed by grain-boundary sliding, whereas coarser-grained films deformed by dislocation slip.

In order to determine the strain-rate sensitivity, ${ }^{30}$ the indenter was first inserted into the sample to a depth of 500 $\mathrm{nm}$; then the load was reduced by $10 \%$ and kept constant. The displacement was then monitored as a function of time for $100 \mathrm{~s}$. Two sets of measurements were conducted, approaching the maximum load prior to measurement at 5 and $20 \mathrm{~nm} / \mathrm{s}$, respectively. In order to obtain $\dot{\varepsilon}$, the results were fitted empirically using the equation 


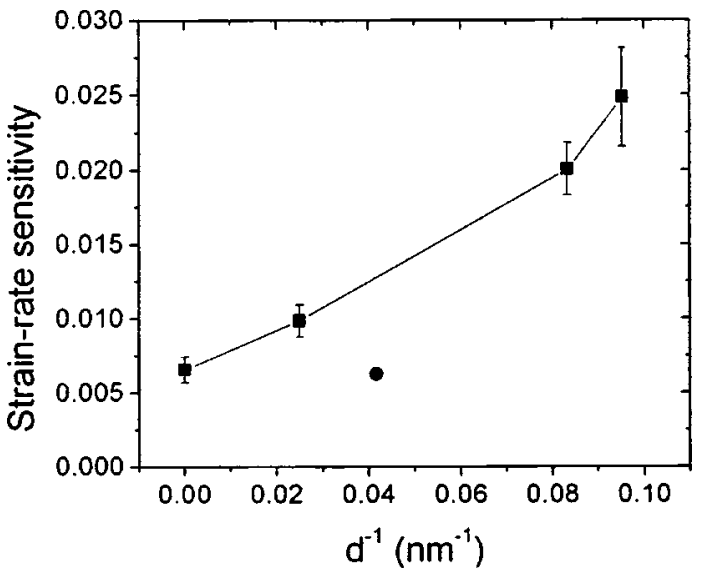

FIG. 4. Strain-rate sensitivity as a function of $d^{-1}$. ( ) strain-rate sensitivity of $30 \mathrm{~nm} \mathrm{Fe}$ : 0.006 ( $\cong$ value for as-received Fe and conventional coarsegrained $\mathrm{Fe})$ (Ref. 38).

$$
h(t)=h_{0}+A \exp \left[-\left(\frac{t}{\tau}\right)^{n}\right]
$$

where $h(t)$ is the displacement as a function of time $t$, and $h_{0}, A, t_{0}, \tau$, and $n$ are constants. The strain rate, stress, and strain-rate sensitivity $m$ were calculated using Eqs. (3), (4), and (2), respectively.

In Fig. 4, the strain-rate sensitivity is displayed as a function of the reciprocal volume-averaged grain size. It is observed to be very small at large grain sizes and to increase monotonically with decreasing grain size. This suggests that a time-dependent deformation mechanism, not based on dislocation slip, is active at room temperature. Grain boundary sliding is a likely mechanism. A similar result was observed by Mayo et al. ${ }^{30}$ in nanophase $\mathrm{TiO}_{2}$. This result is also consistent with our interpretation of the softening observed in Fig. 3. We note that with an indentation size $(\leqslant 500 \mathrm{~nm})$ sufficiently smaller than the particle size $(\geqslant 30 \mu \mathrm{m})$, the measured strain-rate sensitivity is not expected to be affected by interparticle sliding. In fact, the sample with the smallest powder particle size, which would be most susceptible to interparticle sliding, has the smallest strain-rate sensitivity. In addition, the strain-rate sensitivity of this sample which also had the largest grain size, was observed not to be affected by further consolidation. These facts confirm that the strain-rate sensitivity is not affected by the interparticle sliding. Malow et $a l .{ }^{38}$ measured the strain-rate sensitivity of nanocrystalline Fe by an automated ball indentation technique. The values they reported, 0.0067 and 0.0060 at grain sizes of 21 and 24 $\mathrm{nm}$, respectively, were significantly lower than our values at the same grain sizes and comparable to that of our asreceived $\mathrm{Fe}$ and typical coarse-grained $\mathrm{Fe}$. We suggest that grain boundary sliding was reduced in Ref. 38 by impurity redistribution and/or grain boundary relaxation due to the heat treatment.

We finally note that the grain boundary misorientation angle is known to increase with milling time due to grain boundary dislocations created by deformation. ${ }^{39,40}$ The distribution of misorientation angles is expected to affect mechanical properties based on grain boundary sliding, such as hardness and strain-rate sensitivity in nanocrystals. ${ }^{41}$ There- fore, identification of the evolution of grain boundary misorientation with milling time will be necessary for gaining further understanding of the mechanical properties of nanocrystalline Fe.

\section{SUMMARY}

In summary, the hardness of nanocrystalline Fe formed by mechanical attrition was measured at a nanoscale, avoiding artifacts due to porosity or annealing. The Hall-Petch relation was obeyed at grain sizes smaller than previously observed, but a negative slope of the Hall-Petch relation (inverse Hall-Petch relation) was observed at volume-averaged grain sizes below $18 \mathrm{~nm}$. The strain-rate sensitivity was observed to increase with decreasing grain size, possibly due to grain boundary sliding at room temperature.

\section{ACKNOWLEDGMENTS}

This work was funded by the National Science Foundation, Grant No. DMR-9902435. The authors acknowledge useful discussions with Professor A. Ghosh and Professor R. Gibala.

${ }^{1}$ H. Gleiter, Prog. Mater. Sci. 33, 223 (1989).

${ }^{2}$ J. R. Weertman, D. Farkas, K. Hemker, H. Kung, M. Mayo, R. Mitra, and H. Van Swygenhoven, MRS Bull. 24, 44 (1999).

${ }^{3}$ V. Y. Gertsman, M. Hoffmann, H. Gleiter, and R. Birringer, Acta Metall. Mater. 42, 3539 (1994).

${ }^{4}$ H. Chang, C. J. Altstetter, and R. S. Averback, J. Mater. Res. 7, 2962 (1992).

${ }^{5}$ C. C. Koch, Nanostruct. Mater. 9, 13 (1997).

${ }^{6}$ K. Lu, Mater. Sci. Eng., R. 16, 161 (1996).

${ }^{7}$ E. O. Hall, Proc. Phys. Soc. London, Sect. B 64, 747 (1951).

${ }^{8}$ N. J. Petch, J. Iron Steel Inst., London 174, 25 (1953).

${ }^{9}$ R. W. Siegel and G. E. Fougere, Mater. Res. Soc. Symp. Proc. 362, 219 (1995).

${ }^{10}$ D. Tabor, The Hardness of Metals (Clarendon, Oxford, UK, 1951), p. 95.

${ }^{11}$ G. E. Dieter, Mechanical Metallurgy, 3rd ed. (McGraw-Hill, New York, 1986), pp. 325-337.

${ }^{12}$ A. H. Chokshi, A. Rosen, and H. Gleiter, Scr. Metall. 23, 1679 (1989).

${ }^{13}$ G. Palumbo, U. Erb, and K. T. Aust, Scr. Metall. Mater. 24, 2347 (1990).

${ }^{14}$ K. Lu, W. D. Wei, and J. T. Wang, Scr. Metall. Mater. 24, 2319 (1990).

${ }^{15}$ T. Christman and M. Jain, Scr. Metall. Mater. 25, 767 (1991).

${ }^{16}$ D. K. Kim and K. Okazaki, Mater. Sci. Forum 88-90, 553 (1992).

${ }^{17}$ H. Chang, C. J. Altstetter, and R. S. Averback, J. Mater. Res. 7, 2962 (1992).

${ }^{18}$ C. Cheung, G. Palumbo, and U. Erb, Scr. Metall. Mater. 31, 735 (1994).

${ }^{19}$ U. Erb, Nanostruct. Mater. 6, 533 (1995).

${ }^{20}$ H. Alves, M. Ferreira, U. Köster, and B. Müller, Mater. Sci. Forum 225226, 769 (1996).

${ }^{21}$ A. S. Khan, H. Zhang, and L. Takacs, Int. J. Plasticity 16, 1459 (2000).

${ }^{22}$ T. Volpp, E. Göring, W. M. Kuschke, and E. Arzt, Nanostruct. Mater. 7, 855 (1997).

${ }^{23}$ C. C. Koch and J. Narayan, Mater. Res. Soc. Symp. Proc. 634, B5.1.1 (2001).

${ }^{24}$ T. R. Malow and C. C. Koch, Metall. Mater. Trans. A 29A, 2285 (1998).

${ }^{25}$ V. G. Gryaznov, V. A. Solov'ev, and L. I. Trusov, Scr. Metall. Mater. 24, 1529 (1990).

${ }^{26}$ T. G. Nieh and J. Wadsworth, Scr. Metall. Mater. 25, 955 (1991).

${ }^{27}$ M. Ke, S. A. Hackney, W. W. Milligan, and E. C. Aifantis, Nanostruct. Mater. 5, 689 (1995).

${ }^{28}$ J. Schiøtz, F. D. DiTolla, and K. W. Jacobsen, Nature (London) 391, 561 (1998).

${ }^{29}$ H. Van Swygenhoven and A. Caro, Appl. Phys. Lett. 71, 1652 (1997).

${ }^{30}$ M. J. Mayo, R. W. Siegel, A. Narayanasami, and W. D. Nix, J. Mater. Res. 5, 1073 (1990). 
${ }^{31}$ H. H. Tian and M. Atzmon, Philos. Mag. A 79, 1769 (1999).

${ }^{32}$ W. C. Oliver and G. M. Pharr, J. Mater. Res. 7, 1564 (1992).

${ }^{33}$ K. W. McElhaney, J. J. Vlassak, and W. D. Nix, J. Mater. Res. 13, 1300 (1998).

${ }^{34}$ G. Palumbo, S. J. Thorpe, and K. T. Aust, Scr. Metall. 24, 1347 (1990).

${ }^{35}$ H. S. Kim and M. B. Bush, Nanostruct. Mater. 11, 361 (1999).

${ }^{36}$ W. D. Nix and H. Gao, J. Mech. Phys. Solids 46, 411 (1998).
${ }^{37}$ P. Scherrer, Nachrichten Göttinger Gesellschaft 2, 98 (1918)

${ }^{38}$ T. R. Malow, C. C. Koch, P. Q. Miraglia, and K. L. Murty, Mater. Sci. Eng., A 252, 36 (1998).

${ }^{39}$ H. J. Fecht, Nanostruct. Mater. 6, 33 (1995).

${ }^{40}$ H. Van Swygenhoven, P. M. Derlet, and A. Hasnaoui, Phys. Rev. B 66, 024101 (2002).

${ }^{41}$ L. Lu, M. L. Sui, and K. Lu, Science 287, 1463 (2000). 